OPEN ACCESS

Edited by:

Mahmood Yaseen Hachim, Mohammed Bin Rashid University of Medicine and Health Sciences, United Arab Emirates

Reviewed by: Ibrahim Y. Hachim, University of Sharjah, United Arab Emirates

Shroque Zaher,

Mohammed Bin Rashid University of Medicine and Health Sciences,

United Arab Emirates Nikolaj Frost,

Charité-Universitätsmedizin Berlin, Germany

*Correspondence: Hongwen Gao gaohw@jlu.edu.cn Ping-Li Sun pinglisun@naver.com

Specialty section:

This article was submitted to Pulmonary Medicine,

a section of the journal

Frontiers in Medicine

Received: 27 October 2020 Accepted: 30 December 2020 Published: 25 January 2021

Citation:

Yu S, Jia M, Li Y, Sun P-L and Gao H (2021) Differential Expression of $P D-L 1$ in Central and Peripheral and TTF1-Positive and-Negative Small-Cell Lung Cancer.

Front. Med. 7:621838. doi: 10.3389/fmed.2020.621838

\section{Differential Expression of PD-L1 in Central and Peripheral and TTF1-Positive and -Negative Small-Cell Lung Cancer}

\author{
Shili Yu, Meng Jia, Yuemin Li, Ping-Li Sun* and Hongwen Gao* \\ Department of Pathology, The Second Hospital of Jilin University, Changchun, China
}

Background: Central and peripheral location as well as thyroid transcription factor-I (TTF-1) expression was reported to be associated with different characteristics and prognosis of small-cell lung cancer (SCLC). This study aimed to investigate differential expression of PD-L1 in different SCLC subtypes, and in biopsy and resection specimens.

Methods: We retrospectively analyzed 142 SCLC tumor samples using immunohistochemistry to correlate PD-L1 (22C3) expression with clinicopathologic features and survival data.

Results: PD-L1 expression was found in 19.7\% SCLCs (28/142) and was more frequent in females than in males $(32 \%, 16 / 50$ vs. $13 \%, 12 / 92, p=0.009)$, in central type than in peripheral type SCLCs $(26 \%, 26 / 100$ vs. $4.8 \%, 2 / 42, p=0.003)$, and in TTF-1 positive than in negative SCLCs $(23.8 \%, 25 / 105$ vs. $8.1 \%, 3 / 37, p=0.039)$. PD-L1 expression was associated with vascular $(p=0.001)$ and lymphatic invasion $(p=0.001)$. There was no significant difference in PD-L1 expression between biopsy and resection specimens. On univariate analysis, patients with PD-L1 expression had significantly shorter progression-free survival (PFS; $p=0.026$ ) and overall survival (OS; $p=0.012$ ). Multivariate analysis revealed that PD-L1 expression was an independent prognostic factor for OS (HR, 2.317; 95\% Cl 1.199-4.478; $p=0.012)$ and PFS $(H R, 1.636 ; 95 \% \mathrm{Cl}$ $0.990-2.703 ; p=0.051)$ in SCLC.

Conclusions: PD-L1 expression was more frequent in central type, TTF-1 positive SCLCs, and predicted a poor clinical outcome in these patients. Therefore, tumor location and TTF-1 expression could predict expression status of PD-L1, and could potentially serve as clinical response to immunotherapy.

Keywords: small-cell lung cancer, PD-L1, central and peripheral, immunohistochemistry, clone 22C3, TTF-1

\section{INTRODUCTION}

Small-cell lung cancer (SCLC) is an aggressive malignant disease with early development of metastasis. Surgery generally benefits only about $1 \%$ of the patients (1). The first-line therapy for SCLC is platinum-based systemic chemotherapy which has a good initial response rate. However, the vast majority of patients relapse and become resistant within a year (2). Immunotherapy, especially the one based on programmed death 1/programmed death-ligand 1 (PD-1/PD-L1) 
checkpoint inhibitor has generated significant breakthrough in the treatment of SCLC recently $(3,4)$.

The expression of PD-L1 on tumor cells has demonstrated good correlation with response in some clinical trials, but positive responses were only observed in a relatively small section of the patients with PD-L1 expression. Moreover, evidence suggests that patients with $\mathrm{PD}-\mathrm{L} 1-$ negative tumors may also respond to immunotherapy $(2,5,6)$. This has prompted many researchers to seek out the subtype of patients who could benefit from immune treatments that has not yet been identified in SCLC.

The morphological features of small-cell carcinoma are relatively consistent between different tumors and different areas of an individual tumor. However, studies have shown inter-and intratumoral heterogeneity of SCLC, and the impact of these differences on the response to specific therapeutic agents in both patients and animal models (7-10). SCLC tumors have been grouped into distinct subgroups based on a few factors including tumor location, expression of immunohistochemical markers, and genetic profiles. Previous evidence suggest that the central and peripheral type SCLCs show different immunohistochemical phenotypes (TTF-1 and some neuroendocrine markers) and prognosis $(11,12)$. In a mouse model of SCLC, central and peripheral tumors showed different response to cisplatin treatment (7). TTF-1 was expressed in $81-97 \%$ of SCLCs and TTF-1-negative SCLCs exhibited decreased neuroendocrine differentiation, but the prognosis and treatment efficacy of its expression remains unclear because of the limited number of reports $(8,11,13)$. These facts raise the questions as to whether PD-L1 expression differs in different types of SCLC and what may be the prognostic significance of its expression?

Although few studies have evaluated the expression of PDL1 and its prognostic role in SCLC, the results are inconsistent $(14,15)$. No study has evaluated PD-L1 expression in different SCLCs based on tumor location, TTF-1 expression, and specimen types so far. Therefore, to address the above questions, we investigated the expression of PD-L1 in different types of SCLCs in terms of clinicopathologic characteristics and prognosis in this study, with the objective of finding more specific candidates for immunotherapy.

\section{MATERIALS AND METHODS \\ Patients and Data Collection}

In this study, a total of 2,524 SCLC patients (24\%, including 71 resection cases) were selected from 10,458 lung cancer patients who were admitted at The Second Hospital of Jilin University (Jilin, China) between January, 2009 and December, 2018. A total of 142 SCLC patients with follow-up data were finally selected. Patients who had received preoperative neoadjuvant chemotherapy or radiotherapy were excluded in this study. A total of 54 resection cases with medical record were enrolled in this study. The median follow-up period was 21.3 months. The overall 3-year survival rate of the patients was $10.8 \%$, and 5 -year survival was $0 \%$. Smoking status was obtained from the electronic medical records or through telephone surveys. Tumors involving segmental or more proximal bronchi were defined as central type, whereas tumors involving subsegmental or more distal bronchi were defined as peripheral type. A few cases were excluded from the study because of the difficulty in grouping them into the central or peripheral types. This study was approved by the Ethics Committee of The Second Hospital of Jilin University.

\section{Definition of Central- and Peripheral-Type SCLC}

Definition of central-and peripheral-type SCLC based on previous reports $(8,16,17)$, primary tumors involving segmental or more proximal bronchi were defined as central-type tumors. Primary tumors involving subsegmental or more distal bronchi were defined as peripheral-type tumors.

\section{Immunohistochemistry (IHC)}

Immunohistochemistry was performed on formalin-fixed, paraffin-embedded tissue sections using the FDA (Food and Drug Administration) and NMPA (National Medical Products Administration) approved PD-L1 IHC 22C3 pharmDx kit on the Dako Autostainer Link 48 platform according to manufacturer recommendations. $\mathrm{PD}-\mathrm{L} 1$ positivity was defined as membranous staining of any intensity in at least $1 \%$ of the tumor cells and inflammatory cells (tumor associated lymphocytes and macrophages) with more than $1 \%$ membrane and/or cytoplasm staining of any intensity was defined as positive. The slides were examined independently by two observers (P-LS and SY) blinded to both the clinical and pathologic data.

\section{Statistical Analysis}

All statistical analyses were performed using SPSS version 22.0 for Windows (Inc., Chicago, IL). Categorical variables were compared using Fisher's exact test or Chi-square test. KaplanMeier analysis was performed for survival curves, and statistical significance was assessed by the log rank test. To evaluate whether a biomarker was an independent prognostic factor of OS, multivariate analysis using the Cox proportional hazard regression model was performed. All tests were two sided, and $p<0.05$ was considered statistically significant.

\section{RESULTS}

\section{Patient Characteristics}

The clinicopathologic features of the 142 patients are summarized in Table 1. The cohort consisted of 92 (64.8\%) men and $50(35.2 \%)$ women with a median age of 61.72 years (range, 41-87), and included 102 (71.8\%) smokers and 40 (28.2\%) non-smokers. Of the 142 tumor samples obtained, 88 were biopsy and 54 were resection samples, and among these 86 (60.6\%) were limited-stage and 56 (39.4\%) were extensive-stage cases. Further, $26.8 \%$ (38/142) of the patients presented with distant metastatic disease at the time of diagnosis, and the metastatic sites included supraclavicular lymph node, liver, and contralateral lungs among others. Of the 54 resected SCLCs, eight cases were available with paired preoperative bronchoscopic biopsy specimens.

Regarding the tumor location, 100 cases $(70.4 \%)$ were of the central type and $42(29.6 \%)$ cases were of the peripheral type. TTF-1 was expressed in $73.9 \%(105 / 142)$ of the SCLC cases. 
TABLE 1 | PD-L1 status in 142 small-cell lung cancer according to clinicopathologic characteristics.

\begin{tabular}{|c|c|c|c|c|}
\hline \multirow[b]{2}{*}{ Variable } & \multicolumn{3}{|c|}{ PD-L1 expression status } & \multirow[b]{2}{*}{$P$-value } \\
\hline & No. (\%) & $\begin{array}{l}\text { Positive } \\
\text { No. (\%) }\end{array}$ & $\begin{array}{c}\text { Negative } \\
\text { No. (\%) }\end{array}$ & \\
\hline Total & 142 & $28(19.7)$ & $114(80.3)$ & \\
\hline \multicolumn{5}{|l|}{ Age (year) } \\
\hline$<60$ & $67(47.2)$ & $16(23.9)$ & $51(76.1)$ & 0.293 \\
\hline$\geq 60$ & $75(52.8)$ & $12(16.0)$ & $63(84.0)$ & \\
\hline \multicolumn{5}{|l|}{ Sex } \\
\hline Male & $92(64.8)$ & $12(13.0)$ & $80(87.0)$ & 0.009 \\
\hline Female & $50(35.2)$ & $16(32.0)$ & $34(68.0)$ & \\
\hline \multicolumn{5}{|l|}{ Smoking status } \\
\hline Smoker & $102(71.8)$ & 20 (19.6) & $82(80.4)$ & 0.958 \\
\hline Non-smoker & $40(28.2)$ & $8(20.0)$ & $32(80.0)$ & \\
\hline \multicolumn{5}{|l|}{ Specimen types } \\
\hline Biopsy & $88(62.0)$ & $17(19.3)$ & $71(80.7)$ & 1.000 \\
\hline Resection & $54(38.0)$ & $11(20.4)$ & $43(79.6)$ & \\
\hline \multicolumn{5}{|l|}{ Tumor locations } \\
\hline Central-type & $100(70.4)$ & $26(26.0)$ & $74(74.0)$ & 0.003 \\
\hline Peripheral-type & $42(29.6)$ & $2(4.8)$ & 40 (95.2) & \\
\hline \multicolumn{5}{|l|}{ Stage } \\
\hline Limited-stage & $86(60.6)$ & $17(19.8)$ & $69(80.2)$ & 0.985 \\
\hline Extensive-stage & $56(39.4)$ & $11(19.6)$ & $45(80.4)$ & \\
\hline \multicolumn{5}{|l|}{ Pleural invasion } \\
\hline Present & $13(24.1)$ & $2(15.4)$ & $11(84.6)$ & 1.000 \\
\hline Absent & $41(75.9)$ & $9(22.0)$ & $32(78.0)$ & \\
\hline \multicolumn{5}{|l|}{ Vascular invasion } \\
\hline Present & $24(44.4)$ & $10(41.7)$ & 14 (58.3) & 0.001 \\
\hline Absent & 30 (55.6) & $1(3.3)$ & 29 (96.7) & \\
\hline \multicolumn{5}{|l|}{ Lymphatic invasion } \\
\hline Present & $20(37.0)$ & $9(45.0)$ & $11(55.0)$ & 0.001 \\
\hline Absent & $34(63.0)$ & $2(5.9)$ & $32(94.1)$ & \\
\hline \multicolumn{5}{|l|}{ TTF-1 expression } \\
\hline Positive & 105 (73.9) & 25 (23.8) & 80 (76.2) & 0.039 \\
\hline Negative & $37(26.1)$ & $3(8.1)$ & 34 (91.9) & \\
\hline \multicolumn{5}{|l|}{ Chromogranin A } \\
\hline Positive & $115(82.1)$ & 26 (22.6) & $89(77.4)$ & 0.165 \\
\hline Negative & 25 (17.9) & $2(8.0)$ & 23 (92.0) & \\
\hline \multicolumn{5}{|l|}{ Synaptophysin } \\
\hline Positive & $129(92.1)$ & 26 (20.2) & 103 (79.8) & 1.000 \\
\hline Negative & $11(7.9)$ & 2 (18.2) & $9(81.8)$ & \\
\hline \multicolumn{5}{|l|}{ CD56 } \\
\hline Positive & $129(93.5)$ & $26(20.2)$ & $103(79.8)$ & \\
\hline Negative & $9(6.5)$ & $2(22.2)$ & $7(77.8)$ & 1.000 \\
\hline
\end{tabular}

The bold values indicates significantly difference.

Moreover, $72.0 \%(72 / 100)$ of the central type tumors and $78.6 \%$ (33/42) of the peripheral type tumors showed positivity for TTF1. There was no significant correlation between TTF-1 expression and tumor type. Most of the cases showed positive expression of the common neuroendocrine markers including chromogranin A $(82.1 \%, 115 / 140)$, synaptophysin $(92.1 \%, 129 / 140)$, and CD56
(93.5\%, 129/138). The Ki-67 labeling index was high (>70\%) in almost all cases $(99.3 \%, 141 / 142)$.

\section{Expression and Clinicopathologic Correlation of PD-L1 in Total SCLC}

PD-L1 protein expression was not detected in peribronchial mucus glands and bronchial epithelium, non-neoplastic type I pneumocytes, type II pneumocytes, and mesenchymal cells. Some inflammatory cells in the lung cancer microenvironment including $\mathrm{T}$ cells, macrophages, and mast cells showed weak to moderate PD-L1 cytoplasmic and membrane immunoreactivity (Figures 1A-F). PD-L1 expression was detected in the membrane and/or cytoplasm of tumor cells (Figures 1G-I). Tumor cells with more than $1 \%$ membrane staining of any intensity was defined as positive and a total of 19.7\% (28 of 142) of the SCLC cases were found to express PD-L1. Tumor associated lymphocytes and macrophages with more than $1 \%$ membrane and/or cytoplasm staining of any intensity was defined as positive and the total percentage is $41.5 \%(59 / 142)$. The intensity of staining ranged from weak to moderate and strong, and majority of the cases showed moderate staining. The stained tumor percentage of the positive cases was between 2 and $35 \%$ with a mean value of $16 \%$.

Among the 142 patients, PD-L1 expression was more commonly observed in females than in males $(32 \%, 16 / 50$ vs. $13 \%, 12 / 92, p=0.009)$, in central type than in peripheral type SCLC $(26 \%, 26 / 100$ vs. $4.8 \%, 2 / 42, p=0.003)$, and in TTF- 1 positive than in negative cases $(23.8 \%, 25 / 105$ vs. $8.1 \%$, $3 / 37, p=0.039)$. There was a significant correlation between PD-L1 expression and the presence of vascular $(p=0.001)$ and lymphatic $(p=0.001)$ invasion. No significant difference of PD-L1 expression was observed between smokers $(19.6 \%$, $20 / 102)$ and non-smokers $(20.0 \%, 8 / 40)$, biopsy $(19.3 \%, 17 / 88)$ and resected specimens $(20.4 \%, 11 / 54)$, limited-stage $(19.8 \%$, $17 / 86)$, and extensive-stage cases $(19.6 \%, 11 / 56)$. Correlation between PD-L1 expression and neuroendocrine markers was also evaluated and no correlation was found between PD-L1 expression and any of the neuroendocrine markers (Table 1).

\section{PD-L1 Expression in Different Types of SCLCs}

To investigate whether PD-L1 was differentially expressed in different types of SCLCs, we analyzed the association of PD-L1 expression and some clinicopathological parameters including tumor location (central and peripheral), TTF-1 expression (positive and negative), and sample types (biopsy and resection).

There was a significant correlation between PD-L1 expression and female sex $(p=0.035)$, the presence of vascular invasion $(p<0.001)$, the presence of lymphatic invasion $(p=0.004)$, and TTF-1 expression $(p=0.001)$ in central type SCLC. There were no significant correlations between PD-L1 expression and the clinicopathologic characteristics except a negative correlation with TTF-1 expression ( $p=0.042)$ in the peripheral type SCLC (Table 2).

Significant differences in PD-L1 expression were observed between female and male sex $(p=0.035)$, and between central 

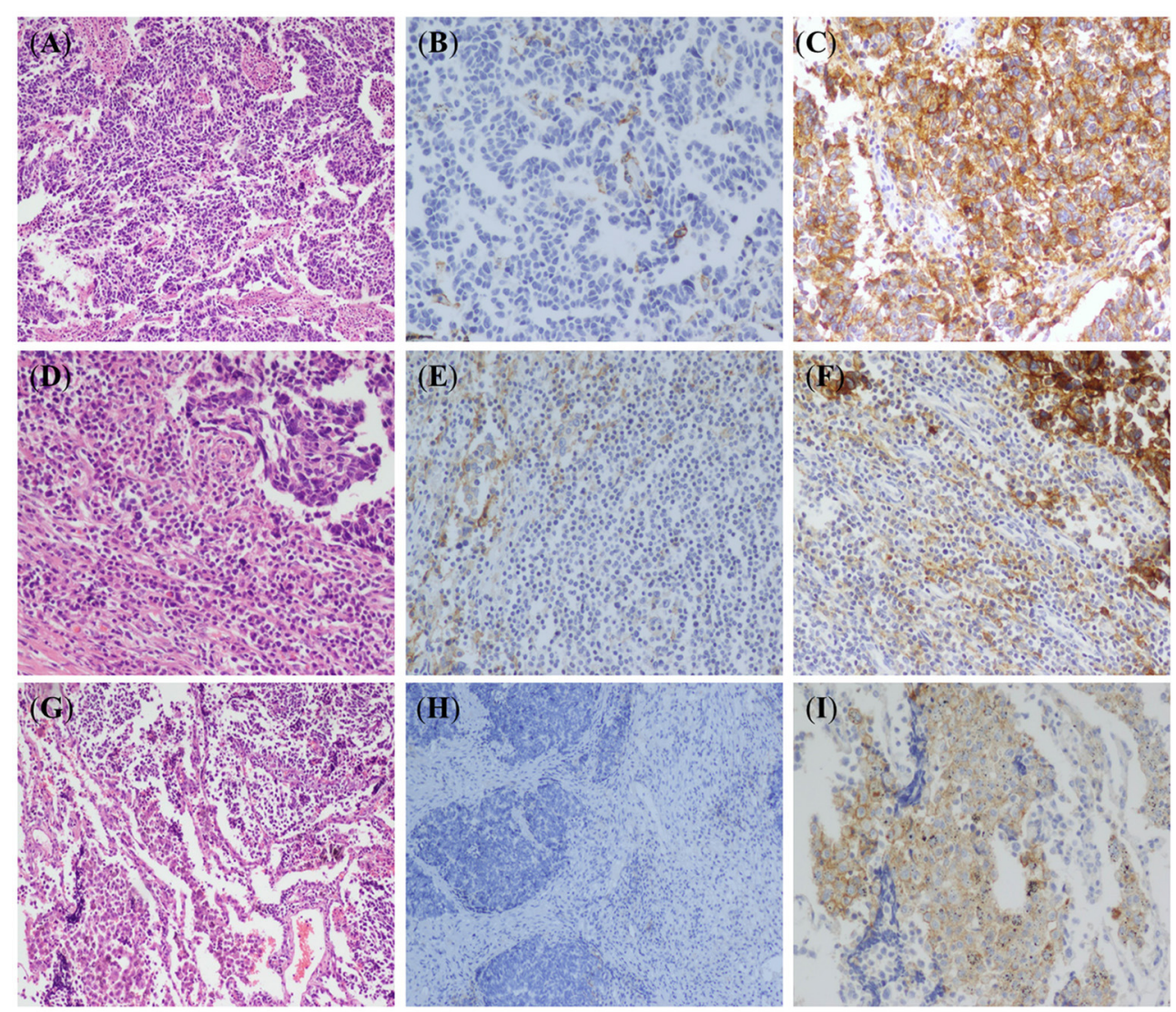

FIGURE 1 | Hematoxylin-eosin (HE) staining and PD-L1 immunohistochemical (IHC) staining in small-cell lung cancer (SCLC). (A) HE stained images of SCLC tumor ( $\times 100)$. (B) IHC stained images of PD-L1 negative in SCLC tumor cells (EnVision, $\times 100)$. (C) IHC stained images of PD-L1 expression in SCLC tumor cells (EnVision, $\times 200)$. (D) Lymphocyte infiltration around tumor cells $(H E, \times 100)$. (E) IHC stained images of PD-L1 negative in tumor infiltrating lymphocytes (EnVision, $\times 100)$. (F) IHC stained images of PD-L1 positive cell membranes of tumor infiltrating lymphocytes (EnVision, $\times 200)$. (G) Macrophages infiltration around tumor cells (HE, $\times 100)$. (H) IHC stained images of PD-L1 negative in tumor-associated macrophages (EnVision, $\times 100)$. (I) IHC stained images of PD-L1 positive cell membranes of tumor-associated macrophages (EnVision, ×200).

and peripheral SCLCs of the TTF-1 positive subtype. PDL1 expression was positively correlated with the presence of vascular invasion $(p=0.003)$ and the presence of lymphatic invasion $(p=0.001)$ in TTF-1 positive SCLCs. Further, there were no significant correlations between PD-L1 expression and any of the clinicopathologic parameters in TTF-1 negative SCLCs (Table 3).

We subsequently evaluated PD-L1 expression in small biopsy and surgically resected specimens (Table 4). Among the 88 small biopsy specimens, PD-L1 expression significantly correlated with TTF-1 $(p=0.023)$ and chromogranin A $(p=0.034)$ expression. In the 54 resected cases, PD-L1 expression was positively correlated with female sex $(p=0.010)$ and central location of the tumor $(p=0.002)$. Of the 54 resected SCLC, eight cases were also available as biopsy specimens for the comparison of PD-L1 expression between small biopsy and surgical resected specimens from the same patients. Of the eight paired cases, PD-L1 expression was observed in one case in which the biopsy and paired resected specimen showed the identical results with the similar intensity and percentage staining of the tumor cells (weak to moderate, 10\% for resection and $8 \%$ for biopsy). The overall concordance rate of PD-L1 expression between resected and their corresponding biopsy specimens was $100 \%$.

\section{Survival Analysis}

Survival analysis was performed among the entire SCLC cohort and then among the different subtypes. In univariate analysis, PD-L1 expression was associated with a significantly poorer PFS and OS ( $p=0.026$ and $p=0.003$, respectively) in entire SCLC cohort (Figures 2A,B). Multivariate Cox analysis confirmed that PD-L1 expression was an independent poor prognostic factor for OS (HR, 2.317; 95\% CI 1.199-4.478; $p=0.012$ ) and tended to be an independent prognostic factor for PFS (HR, 1.636; 95\% CI 0.990-2.703; $p=0.051$ ) (Table 5). In TTF-1 positive and central type SCLCs, PD-L1 expression was associated with poorer PFS $(p=0.014$ and $p=0.042$, respectively) and OS ( $p=0.009$ and $p=0.019$, respectively), and in peripheral type SCLC, with poorer OS $(p=0.005)$. 
TABLE 2 | Correlation between PD-L1 expression and clinicopathologic features in central-type and peripheral-type SCLC.

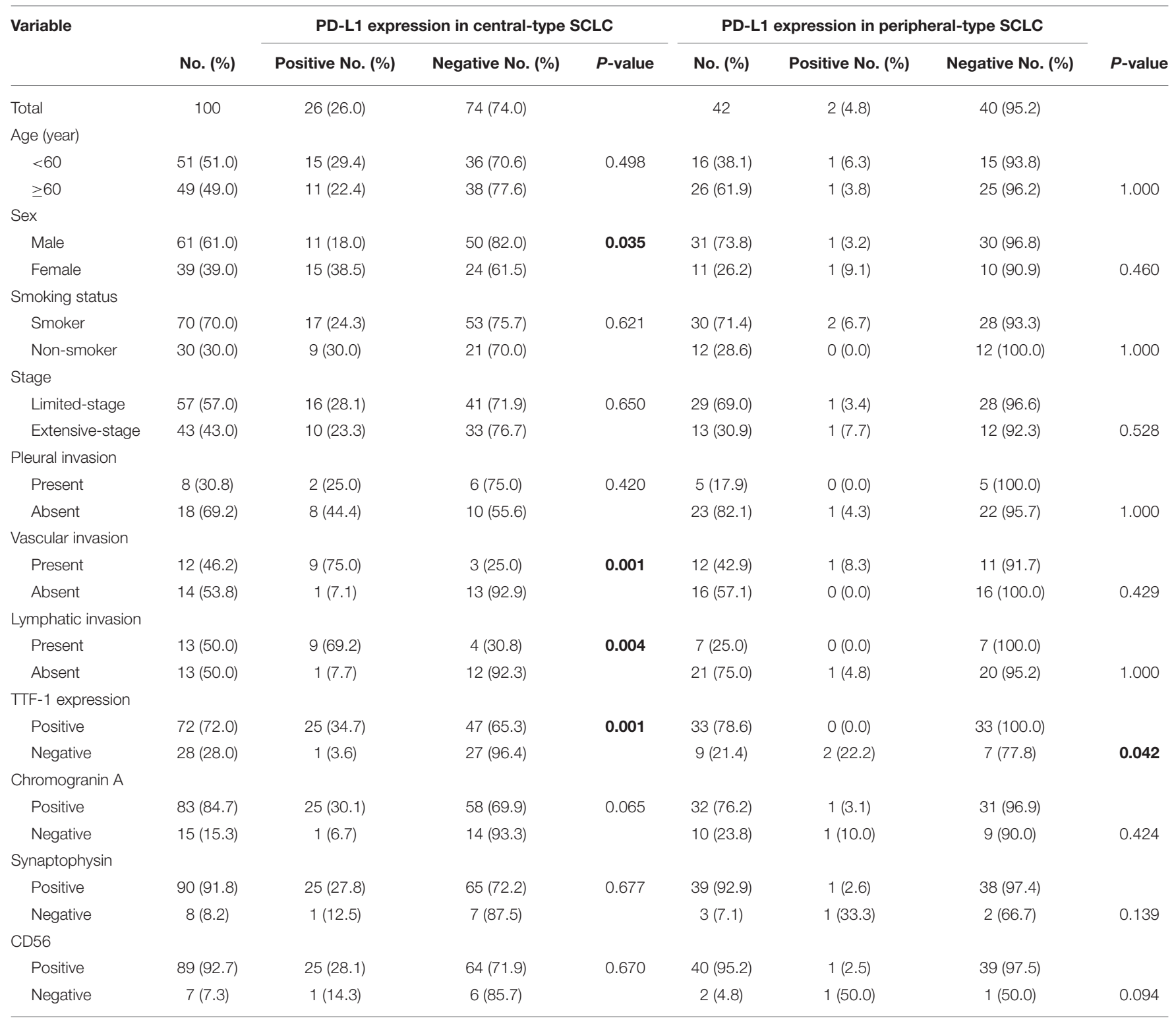

SCLC, small-cell lung cancer. The bold values indicates significantly difference.

\section{DISCUSSION}

To the best of our knowledge, this is the first study to investigate the correlation among tumor location, TTF- 1 expression, and sample type and PD-L1 expression in SCLCs. In this study, the clinicopathologic and prognostic significance of PD-L1 expression were assessed in 142 cases of SCLC and different SCLC subsets. We demonstrated that PD-L1 expression was more frequent in females than males, in central type than peripheral type SCLCs, and in TTF-1 positive than negative SCLCs. PD-L1 expression was positively associated with vascular and lymphatic invasion, which have been implicated in unfavorable prognosis. PD-L1 expression was associated with a significantly poorer PFS and OS in all SCLCs as well as in TTF-1 positive and central type
SCLCs. Multivariate analysis revealed that PD-L1 expression in SCLC was an independent prognostic factor for OS and tended to be an independent prognostic factor for PFS.

So far, there is no study on the relationship between the expression of PD-L1 and gender by using 22C3 antibody in patients with small cell lung cancer. There are some studies on the relationship between the expression of PD-L1 and gender in non-small-cell lung cancer (NSCLC). One of the result showed that there is no significant correlation between gender and the expression of PD-L1 (18). However, the other literature results showed that the expression rate of PD-L1 is higher in male patients (19). Some animal studies and emerging clinical evidence suggested a role for estrogens in upregulation of PD-1 and PDL1 expression, this may be a factor in the high expression level of 
TABLE 3 | Correlation between PD-L1 expression and clinicopathologic features in TTF-1-positive and TTF-1-negative SCLC.

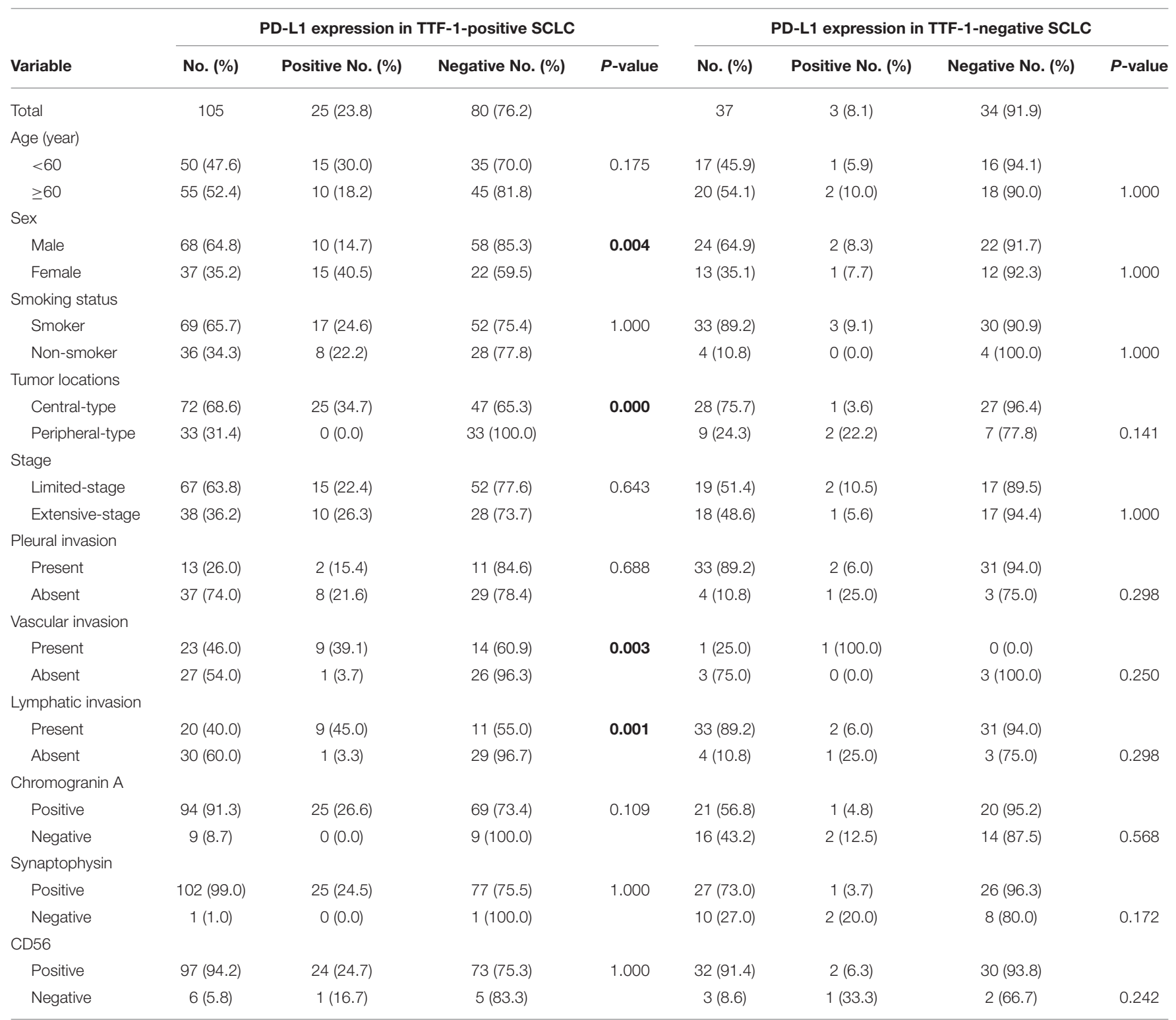

SCLC, small-cell lung cancer. The bold values indicates significantly difference.

$\mathrm{PD}-\mathrm{L} 1$ in women, but further research is needed to confirm the above results $(20,21)$.

Multiple clinical trials have defined PD-L1 as a biomarker for PD-1/PD-L1 checkpoint inhibitor treatment in NSCLC, and PD-L1 is expressed in $\sim 50-70 \%$ of NSCLC as reported in the previous studie (22-25). However, the immunotherapeutic efficacy of PD-L1 in SCLC is far lower than that in NSCLC, and PD-L1 expression is relatively low in SCLC compared to NSCLC. Limited number of studies have evaluated PD-L1 expression in SCLC. PD-L1 expression was found in $19.7 \%$ of SCLCs in our study, and this result is consistent with two previous studies in which the expression rates were 18 and $25 \%$, respectively $(26,27)$. Two other studies showed relatively lower rates of PDL1 expression in SCLCs (2.5 and 10\%), but the sample sizes in those studies were small (39 and 30 , respectively) $(28,29)$. The reason of the variable expression in PD-L1 positivity in our study compared to the other studies may be the use of different types of antibodies as well as the variable definitions of PD-L1 positivity. However, Yasuda et al. (29) used the same antibody and same scoring criteria, they found positivity of only $2.5 \%$. Except for the sample size, the heterogeneity of the study population and interpretation criteria may be responsible for the differences in PD-L1 expression levels. First of all, according to the interpretation criteria, PD-L1 protein expression is determined by using Tumor Proportion Score (TPS), which is the percentage of tumor cells showing partial or complete membrane staining with any extent $(1+-3+)$. Specimens with a $1+$ result in routine immunohistochemical staining are 
TABLE 4 | Correlation between PD-L1 expression and clinicopathologic features in biopsy specimen and resection specimen of small-cell lung cancer.

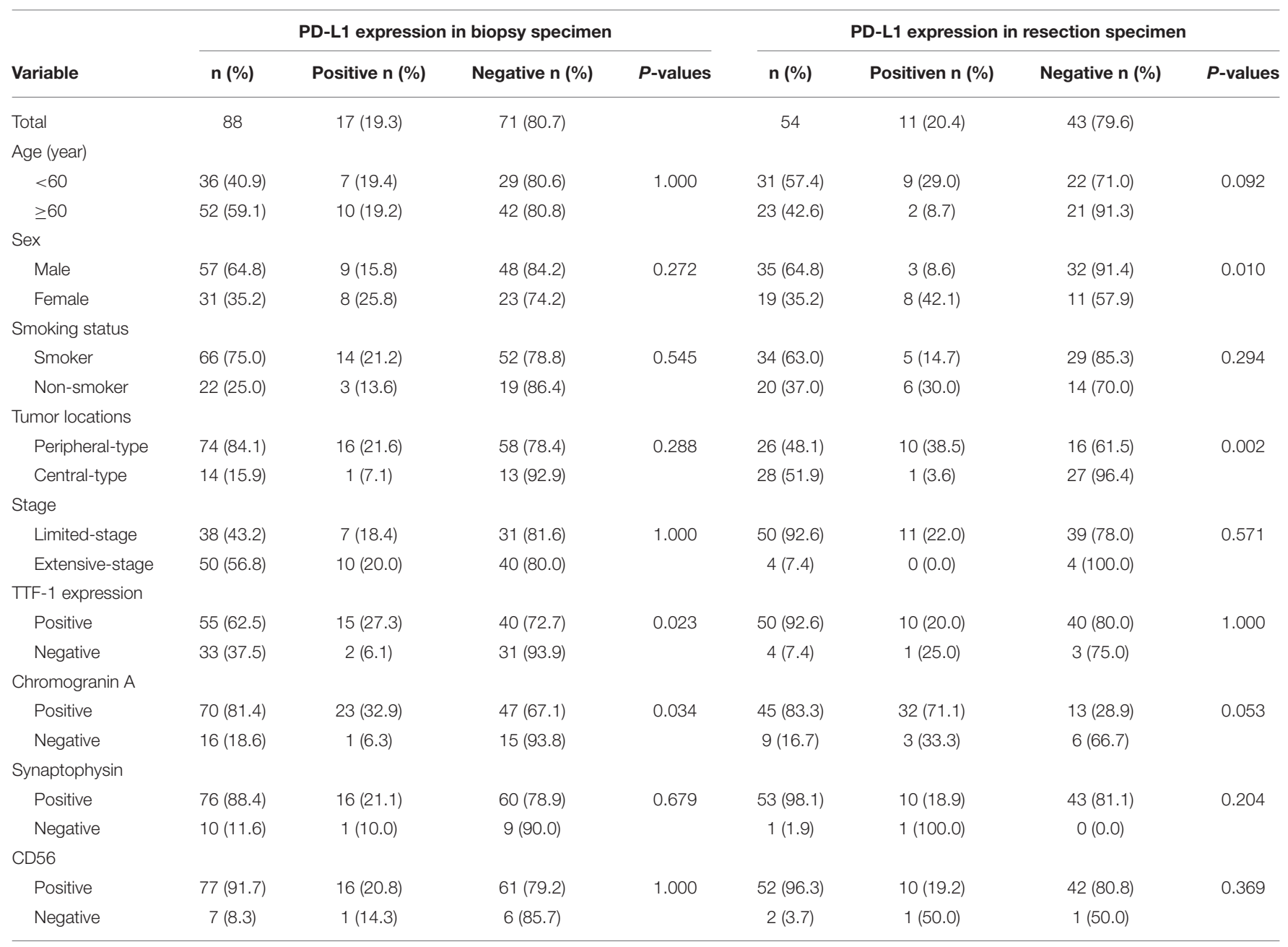

sometimes judged negative, but in the latest criteria, as long as there is a positive cell membrane, it should be judged as positive. All the samples we selected were not treated with chemotherapy, which can avoid the effect of chemotherapy on the expression level of PD-L1, which may also be a reason. Further large-scale investigations or multicenter studies are warranted to determine the actual rate of expression of PD-L1 in SCLCs. PD-L1 was identified as a poor prognostic factor in the current study. The prognostic role of PD-L1 in SCLC is still unclear due to the relatively small number of reports available and the different antibody clones used in the reports $(14,15,30)$. Besides tumor cells, lymphocytes, and macrophages in the stroma were also included in the evaluation in several studies (26) which showed PD-L1 expression rates ranging between 31.7 and 45.7\%, which were similar to the results from our study $(41.5 \%, 59 / 142)(28$, 31). In our study, PD-L1 expression on the inflammatory cells was also analyzed in terms of clinicopathological parameters and prognosis, but no positive correlations were noted.

Although the implementation of anti-PD-1/PD-L1 therapy in SCLC has improved the clinical outcome for a small percentage of patients, the majority of patients show little to no response to the treatment even in cases with high PD-L1 expression. Conversely, clinical benefit from immunotherapeutic agents has also been observed in patients with PD-L1-negative tumors $(5,31)$. These data support the notion that PD-L1 expression cannot be considered as a unique criterion to predict which population will benefit from anti-PD-1 antibodies. Therefore, identification of additional predictors is imperative to improve the response to immune checkpoint inhibitors. Association analysis between the expression of PD-L1 and clinicopathological factors demonstrated high frequency of PD-L1 expression in both central type and TTF-1 expressed SCLC. Primary tumor location has been reported to predict prognosis of SCLC $(12,32)$. A previous study using a mouse model of SCLC reported that centrally located tumors showed better response to cisplatin than peripheral lesions (7). Based on the differential expression of PD-L1, we speculate that peripheral and central SCLCs may show different therapeutic reactivity to immunotherapy. TTF-1 is a master regulator of lung morphogenesis and an important marker for SCLC. TTF-1 expression in SCLC has been reported to be associated with neuroendocrine differentiation and aggressive biology $(8,13)$. However, TTF-1 is not a marker 


\section{A Progression free survival}

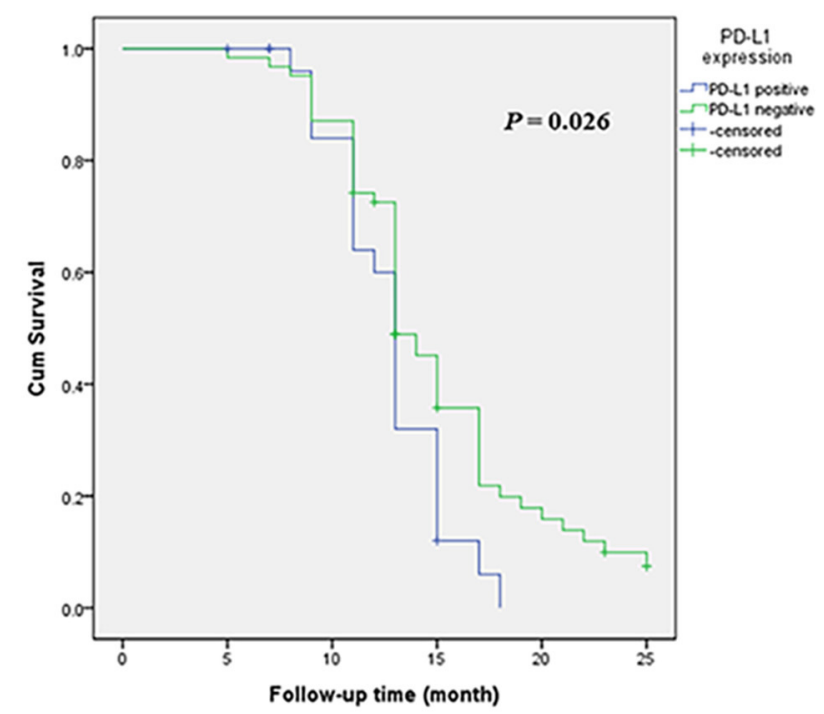

B

\section{Overall survival}

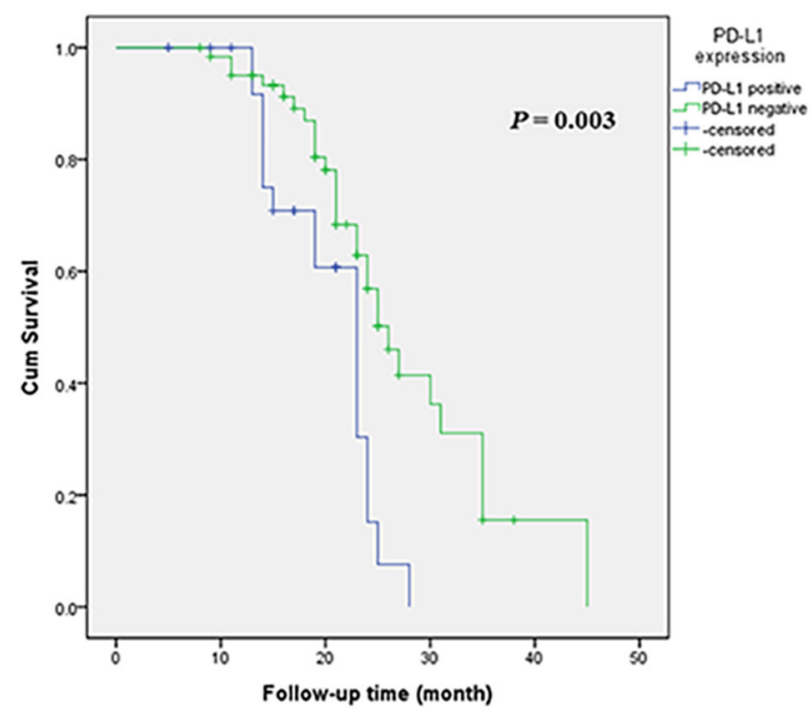

FIGURE 2 | Kaplan-Meier survival estimates of progression-free survival (PFS) and overall survival (OS) based on the expression of PD-L1. (A) Kaplan-Meier plot showing PFS. (B) Kaplan-Meier plot showing OS.

TABLE 5 | Univariate and multivariate analyses of factors associated with PFS and OS in small cell lung cancer patients.

\begin{tabular}{|c|c|c|c|c|c|c|c|c|c|c|c|}
\hline \multirow[t]{3}{*}{ Factor } & \multirow[t]{3}{*}{ Category } & \multicolumn{5}{|c|}{ PFS } & \multicolumn{5}{|c|}{ os } \\
\hline & & \multirow[t]{2}{*}{ Mean } & \multicolumn{2}{|c|}{ Univariate } & \multicolumn{2}{|c|}{ Multivariate } & \multirow[t]{2}{*}{ Mean } & \multicolumn{2}{|c|}{ Univariate } & \multicolumn{2}{|c|}{ Multivariate } \\
\hline & & & $p$ & HR (95\% Cl) & $p$ & HR (95\% Cl) & & $p$ & HR (95\% Cl) & $p$ & HR (95\% Cl) \\
\hline \multirow{2}{*}{$\begin{array}{l}\text { TTF1 } \\
\text { expression }\end{array}$} & Positive vs. & $17.82 \pm 0.54$ & 0.311 & \multirow{2}{*}{$\begin{array}{c}1.259 \\
(0.764-2.075)\end{array}$} & & & $29.35 \pm 1.12$ & \multirow{2}{*}{0.423} & \multirow{2}{*}{$\begin{array}{c}1.300 \\
(0.662-2.552)\end{array}$} & & \\
\hline & Negative & $19.96 \pm 0.65$ & & & & & $32.81 \pm 2.94$ & & & & \\
\hline \multirow{2}{*}{$\begin{array}{l}\text { Vascular } \\
\text { invasion }\end{array}$} & Present vs. & $20.07 \pm 0.81$ & 0.808 & \multirow{2}{*}{$\begin{array}{c}1.066 \\
(0.596-1.908)\end{array}$} & & & $30.96 \pm 1.80$ & \multirow{2}{*}{0.976} & \multirow{2}{*}{$\begin{array}{c}1.011 \\
(1.464-2.204)\end{array}$} & & \\
\hline & Absent & $20.53 \pm 0.91$ & & & & & $32.49 \pm 2.93$ & & & & \\
\hline \multirow{2}{*}{$\begin{array}{l}\text { Lymphatic } \\
\text { invasion }\end{array}$} & Present vs. & $19.55 \pm 0.74$ & 0.188 & \multirow{2}{*}{$\begin{array}{c}1.430 \\
(0.782-2.613)\end{array}$} & & & $29.88 \pm 1.81$ & \multirow{2}{*}{0.377} & \multirow{2}{*}{$\begin{array}{c}1.392 \\
(0.636-3.045)\end{array}$} & & \\
\hline & Absent & $21.45 \pm 0.98$ & & & & & $33.40 \pm 3.03$ & & & & \\
\hline $\begin{array}{l}\text { Pleural } \\
\text { invasion }\end{array}$ & Present vs. & $19.23 \pm 1.16$ & 0.343 & $\begin{array}{c}1.344 \\
(0.674-2.681)\end{array}$ & & & $28.15 \pm 2.56$ & 0.202 & $\begin{array}{c}1.635 \\
(0.715-3.741)\end{array}$ & & \\
\hline Stage & Extensive vs. & $18.08 \pm 0.91$ & 0.051 & 1.745 & 0.087 & 1.566 & $27.04 \pm 1.96$ & 0.072 & 1.020 & 0.265 & 1.450 \\
\hline & Limited & $19.85 \pm 0.58$ & & $(1.060-2.875)$ & & $(0.937-2.616)$ & $31.77 \pm 1.50$ & & $(0.447-2.329)$ & & $(0.754-2.786)$ \\
\hline
\end{tabular}

specific for SCLCs, a significant proportion of extrapulmonary SCLCs are positive for TTF-1. In other studies, TTF-1 has been proposed to be a candidate lineage lung oncogene, knockdown of it reduced growth, and cell viability of lung cancer containing the TTF-1 amplicon (24). A study by Takahashi et al. also demonstrated that TTF-1 stimulated the AKT pathway by directly regulating the expression of a tyrosine kinase-like receptor (22), and AKT pathway has been reported to regulate the expression of PD-L1 in lung cancer (31). The high expression of PD-L1 in TTF-1 positive cases may due to activated oncogenic pathway which was mediated by TTF-1. The positive association between PD-L1 and TTF-1 expression has been reported in pulmonary sarcomatoid carcinomas (33). Furthermore, TTF1 expression was shown to predict the response to immune checkpoint inhibitors in NSCLC (34). So it can be speculated that high TTF-1 expression could be a predictive marker for anti-PD1/PD-L1 therapy in SCLC. Further studies are needed to elucidate this possibility and the underlying mechanisms. 
Discordances between surgically resected and biopsy specimens have been reported in several studies in NSCLC $(35,36)$. However, there is no relevant research in SCLC at present, and this may be because most SCLC cases are unresectable, and only a small number of patients receive surgery owing to the lack of preoperative biopsy results. To investigate whether expressional heterogeneity of PD-L1 exists between biopsy and resection specimens, our study evaluated the PD-L1 expression in the two types of samples and revealed no heterogeneity of PD-L1 expression between the two type of samples. This may due to the relatively lower intratumoral (within a tumor) heterogeneity of SCLCs compared to NSCLCs.

The above findings provide the first evidence of an association between PD-L1 expression, central location, and TTF-1 expression in SCLC. Nevertheless, this study has a few limitations. Firstly, we only included those resected cases that had follow-up data and sufficient specimen, which may have introduced a bias into the analysis. Secondly, the data were collected retrospectively, including some of the information on smoking history which was obtained by telephonic enquiry. It is plausible that some of the patient families may not have provided particularly accurate information, which may explain why there were relatively more nonsmokers in our cohort. Another possible reason is that indoor air pollution or secondhand smoking at home or at work may have contributed to the increased risk of developing lung cancer.

In conclusion, our results revealed a significant association of PD-L1 expression with female sex, central tumor location, TTF-1 expression, and prognosis of the patients with SCLC. Our study provides compelling evidences which can help guide future research and clinical trials in SCLC. However, further studies are needed before drawing definitive conclusions and implementing them clinically.

\section{REFERENCES}

1. Waqar SN, Morgensztern D. Treatment advances in small cell lung cancer (SCLC). Pharmacol Ther Dec. (2017) 180:1623. doi: 10.1016/j.pharmthera.2017.06.002

2. Gazdar AF, Bunn PA, Minna JD. Small-cell lung cancer: what we know, what we need to know and the path forward. Nat Rev Cancer. Dec. (2017) 17:725-37. doi: 10.1038/nrc.2017.87

3. Ott PA, Elez E, Hiret S, Kim DW, Morosky A, Saraf S, et al. Pembrolizumab in patients with extensive-stage small-cell lung cancer: results from the phase Ib KEYNOTE-028 study. J Clin Oncol. (2017) 35:38239. doi: 10.1200/JCO.2017.72.5069

4. Horn L, Mansfield AS, Szczesna A, Havel L, Krzakowski M, Hochmair MJ, et al. First-Line atezolizumab plus chemotherapy in extensive-stage small-cell lung cancer. N Engl J Med. (2018) 379:2220-9. doi: 10.1056/NEJMoa1809064

5. Armstrong SA, Liu SV. Immune checkpoint inhibitors in small cell lung cancer: a partially realized potential. Adv Ther. (2019) 36:182632. doi: $10.1007 / \mathrm{s} 12325-019-01008-2$

6. Gelsomino F, Lamberti G, Parisi C, Casolari L, Melotti B, Sperandi F, et al. The evolving landscape of immunotherapy in small-cell lung cancer: a focus on predictive biomarkers. Cancer Treat Rev. (2019) 79:101887. doi: 10.1016/j.ctrv.2019.08.003

7. Böttger F, Semenova EA, Song JY, Ferone G, van der Vliet J, Cozijnsen $\mathrm{M}$, et al. Tumor heterogeneity underlies differential cisplatin sensitivity

\section{SYNOPSIS}

This study retrospectively analyzed the effect of PD-L1 expression in different types of small-cell lung cancers (SCLCs), in terms of the clinicopathologic features and survival, with the objective of identifying more specific candidates for immunotherapy.

\section{DATA AVAILABILITY STATEMENT}

The original contributions presented in the study are included in the article/supplementary material, further inquiries can be directed to the corresponding author/s.

\section{AUTHOR CONTRIBUTIONS}

P-LS designed the review. SY collected the data and prepared the draft. YL and MJ participated in data interpretation. P-LS and HG provided research fund. All authors read and approved the final manuscript.

\section{FUNDING}

This work was supported by Science and Technology of Jilin Province, Jilin Province Key Laboratory (3D517K363429), The Role and Molecular Mechanism of EMT in the Resistance of ROS1-positive Lung Cancer (20180101014JC/3D518PS23429), Jilin Province Department of Finance Project (3D5197398429, 3D5197464429), and Youth Program of National Natural Science Foundation of China (3A4197642429).

\section{ACKNOWLEDGMENTS}

We would like to thank Xianliang Sha for technical support. in mouse models of small-cell lung cancer. Cell Rep. (2019) 27:334558.e4. doi: 10.1016/j.celrep.2019.05.057

8. Miyauchi E, Motoi N, Ono H, Ninomiya $\mathrm{H}$, Ohyanagi $\mathrm{F}$, Nishio $\mathrm{M}$, et al. Distinct characteristics of small cell lung cancer correlate with central or peripheral origin: subtyping based on location and expression of transcription factor TTF-1. Medicine. (2015) 94:e2324. doi: 10.1097/MD.0000000000002324

9. Park HS, Harder EM, Mancini BR, Decker RH. Central versus peripheral tumor location: influence on survival, local control, and toxicity following stereotactic body radiotherapy for primary non-small-cell lung cancer. J Thorac Oncol. (2015) 10:832-7. doi: 10.1097/JTO.0000000000 000484

10. Shue YT, Lim JS, Sage J. Tumor heterogeneity in small cell lung cancer defined and investigated in pre-clinical mouse models. Transl Lung Cancer Res. (2018) 7:21-31. doi: 10.21037/tlcr.2018.01.15

11. Ito M, Yamashita Y, Miyata Y, Ohara M, Tsutani Y, Ikeda T, et al. Prognostic impact of the primary tumor location based on the hilar structures in nonsmall cell lung cancer with mediastinal lymph node metastasis. Lung Cancer. (2012) 76:93-7. doi: 10.1016/j.lungcan.2011.07.015

12. Kanaji N, Sakai K, Ueda Y, Miyawaki H, Ishii T, Watanabe N, et al. Peripheral-type small cell lung cancer is associated with better survival and higher frequency of interstitial lung disease. Lung Cancer. (2017) 108:12633. doi: 10.1016/j.lungcan.2017.03.013

13. Iida Y, Masuda S, Nakanishi Y, Shimizu T, Nishimaki H, Takahashi $\mathrm{M}$, et al. Clinicopathological characteristics of thyroid transcription 
factor 1-negative small cell lung cancers. Hum Pathol. (2018) 79:12734. doi: 10.1016/j.humpath.2018.05.009

14. Inamura K, Yokouchi Y, Kobayashi M, Ninomiya H, Sakakibara R, Nishio M, et al. Relationship of tumor PD-L1 (CD274) expression with lower mortality in lung high-grade neuroendocrine tumor. Cancer Med. (2017) 6:2347-56. doi: 10.1002/cam4.1172

15. Chang YL, Yang CY, Huang YL, Wu CT, Yang PC. High PD-L1 expression is associated with stage IV disease and poorer overall survival in 186 cases of small cell lung cancers. Oncotarget. (2017) 8:1802130. doi: 10.18632 /oncotarget. 14935

16. Bandoh S, Fujita J, Ueda Y, Fukunaga Y, Dohmoto K, Hojo S, et al. Expression of carcinoembryonic antigen in peripheral- or central-located small cell lung cancer: its clinical significance. Jpn J Clin Oncol. (2001) 31:305-10. doi: 10.1093/jjco/hye067

17. Nobashi T, Koyasu S, Nakamoto Y, Kubo T, Ishimori T, Kim YH, et al. Prognostic value of fluorine-18 fludeoxyglucose positron emission tomography parameters differs according to primary tumour location in small-cell lung cancer. $\mathrm{Br} J$ Radiol. (2016) 89:20150618. doi: 10.1259/bjr.20150618

18. Pan ZK, Ye F, Wu X, An HX, Wu JX. Clinicopathological and prognostic significance of programmed cell death ligand $1(\mathrm{PD}-\mathrm{L} 1)$ expression in patients with non-small cell lung cancer: a meta-analysis. J Thorac Dis. (2015) 7:46270. doi: $10.3978 / j$.issn.2072-1439.2015.02.13

19. Zhang M, Li G, Wang Y, Wang Y, Zhao S, Haihong P, et al. PD-L1 expression in lung cancer and its correlation with driver mutations: a meta-analysis. Sci Rep. (2017) 7:10255. doi: 10.1038/s41598-017-10925-7

20. Polanczyk MJ, Hopke C, Vandenbark AA, Offner H. Estrogen-mediated immunomodulation involves reduced activation of effector $\mathrm{T}$ cells, potentiation of Treg cells, and enhanced expression of the PD- 1 costimulatory pathway. J Neurosci Res. (2006) 84:370-8. doi: 10.1002/jnr.20881

21. Wang C, Dehghani B, Li Y, Kaler LJ, Vandenbark AA, Offner H. Oestrogen modulates experimental autoimmune encephalomyelitis and interleukin17 production via programmed death 1 . Immunology. (2009) 126:32935. doi: 10.1111/j.1365-2567.2008.03051.x

22. Yamaguchi T, Yanagisawa K, Sugiyama R, Hosono Y, Shimada Y, Arima C, et al. NKX2-1/TITF1/TTF-1-Induced ROR1 is required to sustain EGFR survival signaling in lung adenocarcinoma. Cancer Cell. (2012) 21:34861. doi: $10.1016 /$ j.ccr.2012.02.008

23. Gandhi L, Rodríguez-Abreu D, Gadgeel S, Esteban E, Felip E, De Angelis F, et al. Pembrolizumab plus chemotherapy in metastatic non-small-cell lung cancer. N Engl J Med. (2018) 378:2078-92. doi: 10.1056/NEJMoa1801005

24. Kwei KA, Kim YH, Girard L, Kao J, Pacyna-Gengelbach M, Salari K, et al. Genomic profiling identifies TITF1 as a lineage-specific oncogene amplified in lung cancer. Oncogene. (2008) 27:3635-40. doi: 10.1038/sj.onc.1211012

25. Paz-Ares L, Luft A, Vicente D, Tafreshi A, Gümüş M, Mazières J, et al. Pembrolizumab plus chemotherapy for squamous non-small-cell lung cancer. N Engl J Med. (2018) 379:2040-51. doi: 10.1056/NEJMoa1810865

26. Bonanno L, Pavan A, Dieci MV, Di Liso E, Schiavon M, Comacchio $\mathrm{G}$, et al. The role of immune microenvironment in small-cell lung cancer: distribution of PD-L1 expression and prognostic role of
FOXP3-positive tumour infiltrating lymphocytes. Eur J Cancer. (2018) 101:191-200. doi: 10.1016/j.ejca.2018.06.023

27. Yoshimura A, Yamada T, Miyagawa-Hayashino A, Sonobe Y, Imabayashi T, Yamada T, et al. Comparing three different anti-PD-L1 antibodies for immunohistochemical evaluation of small cell lung cancer. Lung Cancer. (2019) 137:108-12. doi: 10.1016/j.lungcan.2019.09.012

28. Gadgeel SM, Pennell NA, Fidler MJ, Halmos B, Bonomi P, Stevenson J, et al Phase II study of maintenance pembrolizumab in patients with extensivestage small cell lung cancer (SCLC). J Thorac Oncol. (2018) 13:13939. doi: 10.1016/j.jtho.2018.05.002

29. Yasuda Y, Ozasa H, Kim YH. PD-L1 expression in small cell lung cancer. $J$ Thorac Oncol. (2018) 13:e40-1. doi: 10.1016/j.jtho.2017.10.013

30. Xu Y, Cui G, Jiang Z, Li N, Zhang X. Survival analysis with regard to PD-L1 and CD155 expression in human small cell lung cancer and a comparison with associated receptors. Oncol Lett. (2019) 17:2960-8. doi: 10.3892/ol.2019.9910

31. Han Y, Liu D, Li L. PD-1/PD-L1 pathway: current researches in cancer. Am J Cancer Res. (2020) 10:727-42.

32. Liu S, Zhang G, Li C, Chen X, Wang S, Wang M, et al. Prognostic factors and survival of patients with small cell lung cancer in a northeastern Chinese population. Thorac Cancer. (2013) 4:143-52. doi: 10.1111/j.1759-7714.2012.00149.x

33. Vieira T, Antoine M, Hamard C, Fallet V, Duruisseaux M, Rabbe N, et al Sarcomatoid lung carcinomas show high levels of programmed death ligand-1 (PD-L1) and strong immune-cell infiltration by TCD3 cells and macrophages. Lung Cancer. (2016) 98:51-8. doi: 10.1016/j.lungcan.2016.05.013

34. Baraibar I, Roman M, Castañón E, García MÁ, Rolfo C, López-Picazo $\mathrm{JM}$, et al. Lymphocytes and neutrophils count after two cycles and TTF1 expression as early outcome predictors during immunotherapy (IT) in stage IV non-small cell lung cancer (NSCLC) patients. J Clin Oncol. (2017) 35:e20553. doi: 10.1200/JCO.2017.35.15_suppl.e20553

35. Ilie M, Long-Mira E, Bence C, Butori C, Lassalle S, Bouhlel L, et al Comparative study of the PD-L1 status between surgically resected specimens and matched biopsies of NSCLC patients reveal major discordances: a potential issue for anti-PD-L1 therapeutic strategies. Ann Oncol. (2016) 27:147-53. doi: 10.1093/annonc/mdv489

36. Jin Y, Shen X, Pan Y, Zheng Q, Chen H, Hu H, et al. Correlation between PDL1 expressionand clinicopathological characteristics of non-small cell lung cancer: a real-world study of a large Chinese cohort. J Thorac Dis. (2019) 11:4591-601. doi: 10.21037/jtd.2019.10.80

Conflict of Interest: The authors declare that the research was conducted in the absence of any commercial or financial relationships that could be construed as a potential conflict of interest.

Copyright $\odot 2021 \mathrm{Yu}$, Jia, Li, Sun and Gao. This is an open-access article distributed under the terms of the Creative Commons Attribution License (CC BY). The use, distribution or reproduction in other forums is permitted, provided the original author(s) and the copyright owner(s) are credited and that the original publication in this journal is cited, in accordance with accepted academic practice. No use, distribution or reproduction is permitted which does not comply with these terms. 